\title{
ARTICLE
}

Received 30 Jan 2015 | Accepted 1 May 2015 | Published 10 Jun 2015

\section{Ligand regulation of a constitutively dimeric EGF receptor}

Daniel M. Freed ${ }^{1}$, Diego Alvarado ${ }^{1, \dagger} \&$ Mark A. Lemmon ${ }^{1}$

Ligand-induced receptor dimerization has traditionally been viewed as the key event in transmembrane signalling by epidermal growth factor receptors (EGFRs). Here we show that the Caenorhabditis elegans EGFR orthologue LET-23 is constitutively dimeric, yet responds to its ligand LIN-3 without changing oligomerization state. SAXS and mutational analyses further reveal that the preformed dimer of the LET-23 extracellular region is mediated by its domain II dimerization arm and resembles other EGFR extracellular dimers seen in structural studies. Binding of LIN-3 induces only minor structural rearrangements in the LET-23 dimer to promote signalling. Our results therefore argue that EGFR can be regulated by allosteric changes within an existing receptor dimer-resembling signalling by insulin receptor family members, which share similar extracellular domain compositions but form covalent dimers.

\footnotetext{
${ }^{1}$ Department of Biochemistry and Biophysics, University of Pennsylvania Perelman School of Medicine, 322A Clinical Research Building, 415 Curie Boulevard, Philadelphia, Pennsylvania 19104-6059, USA. † Present address: Kolltan Pharmaceuticals, New Haven, Connecticut 06511, USA. Correspondence and requests for materials should be addressed to M.A.L. (email: mlemmon@mail.med.upenn.edu).
} 
R eceptor tyrosine kinases (RTKs) control many cellular processes and play causative roles in diseases such as cancer, where they are important therapeutic targets ${ }^{1}$. Early work with the epidermal growth factor (EGF) and plateletderived growth factor (PDGF) receptors established that these RTKs signal as dimers, and further suggested that signalling requires ligand-induced receptor dimerization ${ }^{2,3}$. Several biochemical and structural studies of EGF receptor (EGFR) have subsequently argued that the extra- and intracellular regions of the receptor are structurally independent and flexibly linked ${ }^{4-}$ 7 - consistent with models in which dimerization itself (induced by ligand binding) is the key event in receptor activation. On the other hand, reports that many RTKs can dimerize without ligand (forming inactive 'preformed dimers') ${ }^{8}$ argue that the signalling mechanism cannot be this simple-as does the fact that RTKs from the insulin receptor (IR) family are covalently linked dimers ${ }^{9}$. Structural analysis of IR family members has provided valuable insight into how these constitutively dimeric RTKs are regulated by their ligands ${ }^{10-13}$, but whether (and how) RTKs that are not disulfide-linked dimers are also allosterically regulated through analogous mechanisms remains unclear. Indeed, the precise nature (or oligomerization state) of a 'preformed RTK dimer' has not been described outside the IR family.

In studying EGFR orthologues from different phyla, we discovered that the isolated extracellular region of the C. elegans $\mathrm{EGFR}^{14}$, called LET-23, dimerizes strongly-with a sub-micromolar dissociation constant $\left(K_{\mathrm{d}}^{\mathrm{dim}}\right)$-regardless of the presence of its EGF-like ligand (LIN-3 (ref. 15)). By contrast, the unliganded extracellular region of human EGFR is exclusively monomeric in solution $\left(K_{\mathrm{d}}^{\mathrm{dim}}>1 \mathrm{mM}^{16}\right)$ and that from Drosophila melanogaster EGFR is predominantly monomeric $\left(K_{\mathrm{d}}^{\mathrm{dim}} \sim 40 \mu \mathrm{M}^{17}\right)$-despite the fact that the three extracellular regions share $29 \%-39 \%$ pairwise sequence identity (Supplementary Fig. 1). LET-23 therefore provides a unique opportunity to study the extracellular region of a preformed EGFR dimer. Using biophysical and biochemical approaches, we show that ligand-induced changes in this preformed dimer are relatively small in scale, indicating that EGFR signalling can be regulated by relatively subtle allosteric changes within a receptor dimer. These findings in turn suggest mechanistic similarities between EGFR and the constitutively dimeric IR-family receptors, which share the same domain types over much of their extracellular regions (Fig. 1a).

\section{Results}

The LET-23 extracellular region is constitutively dimeric. During initial purification, we found that-unlike other EGFR extracellular regions ${ }^{16,18}$ - the unliganded LET-23 extracellular region (sLET-23) migrates solely as a dimer in size-exclusion chromatography, and that this behaviour is retained when the invertebrate-specific domain V (Fig. 1a) is deleted to yield a

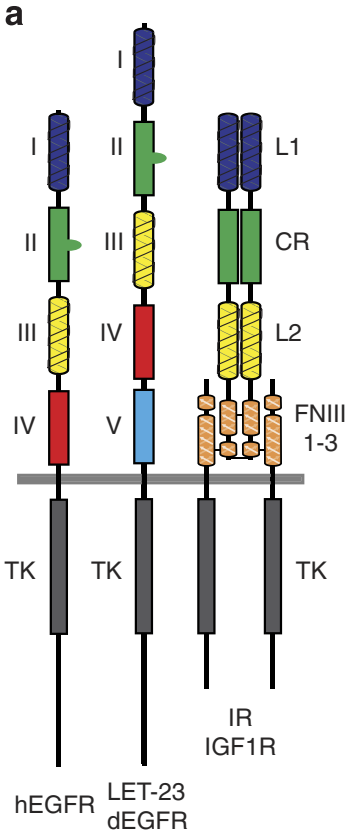

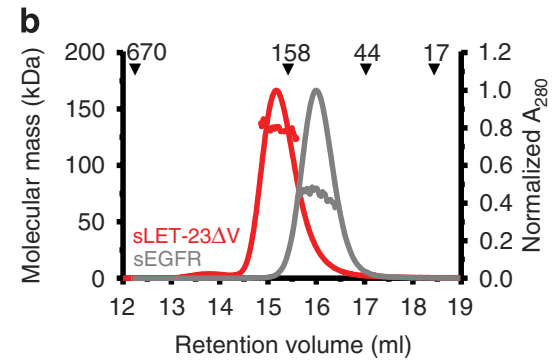

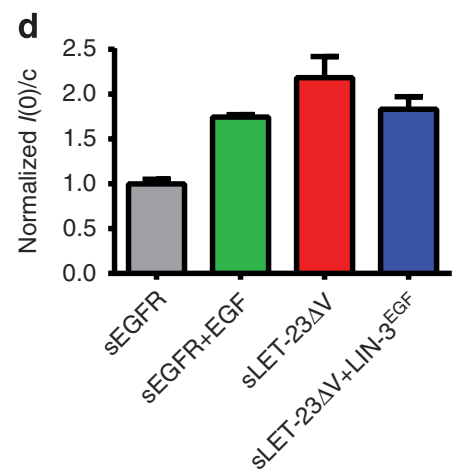

C
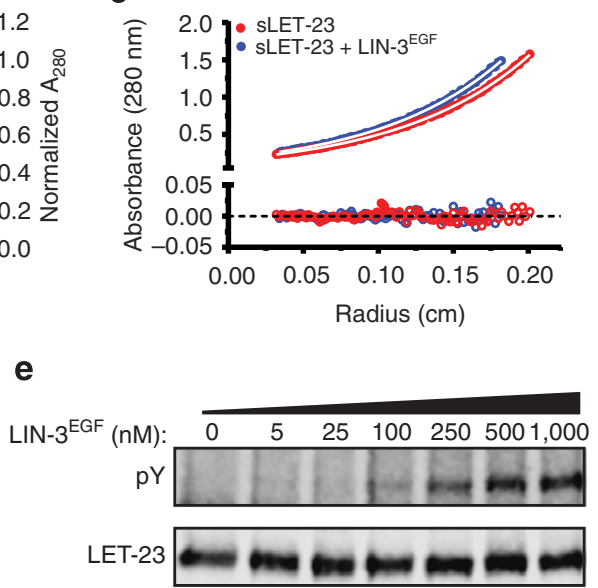

Figure 1 | LET-23 is a ligand-regulated dimer. (a) Domain compositions of human EGFR (hEGFR), C. elegans LET-23 and D. melanogaster EGFR (dEGFR), and members of the covalently dimerized IR family. Domains I and III (L1 and L2 in IR) are $\beta$-helix/solenoid domains. Domains II, IV and V (CR in IR) are cysteine rich. IR also has fibronectin type III (FNIII) domains. Intracellular tyrosine kinase (TK) domains are marked. Note the dimerization arm emanating from domain II in the EGFRs. (b) Size-exclusion chromatography-MALS (SEC-MALS) data for sLET-23AV (red) and human sEGFR (grey) at eluted concentrations of $\sim 1 \mu \mathrm{M}$. Circles denote molecular masses determined by in-line MALS (left axis). Including glycosylation, the monomeric molecular

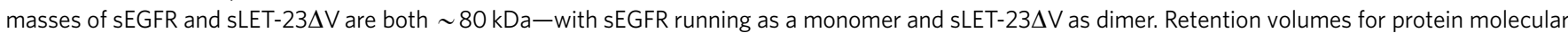
mass standards are marked (with kDa values) across the top of the figure. (c) Representative SE-AUC data for sLET-23 (8 $\mu$ M, 8,200 r.p.m.). Without ligand (red circles), the data across all repeats fit well to a single ideal $190 \pm 22 \mathrm{kDa}$ species-approximately twice the monomeric mass ( $\sim 91 \mathrm{kDa}$ ). In the presence of $60 \mu \mathrm{M} \mathrm{LIN-3} 3^{\mathrm{EGF}}$ (blue circles), the fit is essentially unchanged, yielding a molecular mass of $185 \pm 6 \mathrm{kDa}$ across all repeats (see Table 1). Fits to the data are shown as white curves superimposed on the data points and residuals are shown below the fits as open circles. (d) SAXS-derived normalized $I(0) / c$ values for sEGFR and sLET-23 $\Delta \mathrm{V}$, with and without bound ligand (added at 61-110 $\mu \mathrm{M}$ ) at pH 8, using home-source data. The average $I(0) / \mathrm{c}$ for unliganded (monomeric) sEGFR was set to a relative value of 1.0 and all other $I(0) / c$ values were normalized to this value, with mean \pm s.d. presented for 3-14 independent measurements. (e) Western blotting showing LIN-3 ${ }^{\mathrm{EGF}}$-induced phosphorylation of full-length LET-23 in D. melanogaster S2 cells. The uncropped gel and molecular weight markers are shown in Supplementary Fig. 2. 


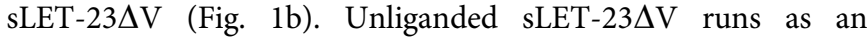
$\sim 140 \mathrm{kDa}$ dimer (compared with a predicted monomeric mass of $71 \mathrm{kDa}$-as also confirmed by in-line multi-angle light scattering (MALS). By contrast, Fig. $1 \mathrm{~b}$ shows that the unliganded human EGFR extracellular region (sEGFR) runs true to its monomeric molecular mass $(\sim 80 \mathrm{kDa})$. Strong dimerization of sLET-23 (including domain V) was also evident in sedimentation equilibrium analytical ultracentrifugation (SE-AUC) studies (Fig. 1c), which detected a single species with a molecular mass of $190 \pm 22 \mathrm{kDa}$ (compared with the expected monomer mass of $91 \mathrm{kDa})$. Importantly, SE-AUC studies revealed that adding excess ligand (LIN-3 ${ }^{\mathrm{EGF}}$ : the purified EGF domain from LIN-3, added at $60 \mu \mathrm{M})$ did not alter the oligomerization state of sLET-23 (Fig. 1c and Table 1), despite binding with an affinity that ensures saturation of the receptor under the conditions of this experiment $\left(K_{\mathrm{d}}=2-4 \mu \mathrm{M}\right.$; Supplementary Fig. 2a,b). Small-angle X-ray scattering (SAXS) studies gave the same result (Fig. 1d and Supplementary Fig. 4). SAXS provides a shape-independent measure of the weightaveraged molecular mass of the scattering species through the parameter $I(0) / \mathrm{c}$, which is the concentration-normalized scattering intensity extrapolated to zero angle. For sEGFR, $I(0) /$ c nearly doubles on addition of EGF (Fig. 1d), as we have reported previously ${ }^{16}$. As unliganded sLET- $23 \Delta \mathrm{V}$ is dimeric, its measured $I(0) / c$ value is approximately twice that of unliganded human sEGFR and approximates $I(0) / c$ for the EGF-induced sEGFR dimer. Importantly, adding excess LIN-3 ${ }^{\text {EGF }}$ does not increase $I(0) / \mathrm{c}$ for sLET-23 (Fig. 1d, see also Supplementary Fig. 4). Taken together, these results reveal that the isolated LET23 extracellular region exists as a dimer, with no change in oligomerization state on association with its activating ligand.

LIN-3 activates dimeric LET-23 in cells. Our biophysical studies show that sLET-23 is entirely dimeric at concentrations as low as $1 \mu \mathrm{M}$, arguing that the isolated LET-23 extracellular region dimerizes with a sub-micromolar $K_{\mathrm{d}}^{\mathrm{dim}}$ in the absence of ligand. The $K_{\mathrm{d}}^{\text {dim }}$ value for unliganded sLET-23 is therefore actually smaller (stronger) than $K_{\mathrm{d}}^{\text {dim }}$ values measured for EGF-bound human sEGFR $\left(\sim 3.3 \mu \mathrm{M}^{16}\right)$, which dimerizes in cell membranes. As the local concentration of EGFRs in a cell is typically in the 1 to $10 \mu \mathrm{M}$ range $\mathrm{e}^{16,19}$, our results imply that LET-23 is constitutively dimerized on the cell surface through interactions driven by its extracellular region alone. However, our findings almost certainly underestimate the strength of LET-23

\begin{tabular}{|c|c|c|}
\hline Receptor & Mass $^{\text {pred }}(\mathrm{kDa})$ & Mass $^{\text {fit }}$ (kDa) \\
\hline sLET-23 & 91 & $190 \pm 22$ \\
\hline sLET-23 + LIN-3 $3^{\text {EGF }}$ & 98 & $185 \pm 6$ \\
\hline 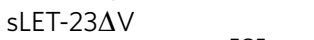 & 71 & $153 \pm 27$ \\
\hline 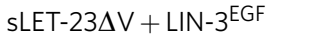 & 78 & $141 \pm 3$ \\
\hline sLET-23AIV/V & 60 & $82 \pm 8$ \\
\hline sLET-23AIV/V + LIN-3 EGF & 67 & $123 \pm 8$ \\
\hline sLET-23 dim-arm & 91 & $94 \pm 7$ \\
\hline
\end{tabular}

SE-AUC, sedimentation equilibrium analytical ultracentrifugation.

Mass ${ }^{\text {pred }}$ is the polypeptide molecular mass of the monomeric protein, as predicted from the primary sequence. Mass $s^{\text {fit }}$ corresponds to the molecular mass obtained by fitting the

experimental data at three speeds for each variant to a single species (for which residuals were small and random). Average Mass ${ }^{\text {fit }}$ values at a concentration of $8 \mu \mathrm{M}$ are shown for sLET-23AIV/ $V$ : all other receptor variants were fully dimeric (or monomeric in the case of SLET-23dim-arm) at all , all or concentrations tested (from 1 to $8 \mu M$ ) and Mass values are quoted as the concentrationindependent average ( \pm s.d.). SLET-23 contains six predicted $\mathrm{N}$-glycosylation sites compared with nine for human sEGFR (which is $\sim 20 \%$ carbohydrate by mass and $\sim 71 \mathrm{kDa}$ polypeptide). We therefore estimate that SLET-23 is $\sim 10 \%$ carbohydrate by mass. Estimates for sLET-23AV and sLET-23AIV/V are $\sim 9 \%$ (4 sites) and $\sim 11 \%$ (4 sites), respectively. See Supplementary Fig. 3 for representative data fits. dimerization at the cell surface. First, we do not account for contributions to dimerization from the transmembrane, juxtamembrane or intracellular domains-which all appear to contribute to human EGFR dimerization ${ }^{7,20-22}$. Second, our argument does not consider pre-orientation effects that will be experienced by LET-23 in the confines of a membrane, which are estimated to further enhance dimerization by up to $10^{6}$-fold ${ }^{23}$

Despite probably being completely dimeric in the cell membrane, LET-23 activation remains strictly dependent on addition of LIN-3 (Fig. 1e and Supplementary Fig. 2c,d). We expressed full-length LET-23 in D. melanogaster Schneider-2 (S2) cells as a null background and showed that LIN $-3^{\mathrm{EGF}}$ is sufficient to activate the heterologously expressed receptor. These first biochemical data for LIN-3/LET-23 also reveal that LIN-3, which is the only LET-23 ligand in C. elegans, resembles one of the lowaffinity EGFR ligands in humans (amphiregulin, epigen and epiregulin ${ }^{24}$ ) or D. melanogaster $\left(\right.$ vein $^{25}$ ). As with other known low-affinity ligands, LIN-3 shows both $\mathrm{ED}_{50}$ values for receptor activation (Fig. 1e and Supplementary Fig. $2 c, d$ ) and $K_{\mathrm{d}}$ values for LET-23 binding (Supplementary Fig. 2a,b) that are 10- to 100-fold higher (weaker) than typically reported for EGF or transforming growth factor- $\alpha$ in humans ${ }^{24}$ (or spitz in D. melanogaster ${ }^{18}$ ).

Structural determinants of sLET-23 dimerization. Our results argue that EGFR signalling in C. elegans is regulated by ligandinduced structural rearrangements within receptor dimers rather than by ligand-induced changes in oligomerization, as is more commonly supposed for this receptor family. These findings also provide an opportunity to assess the structural determinants of constitutive sLET-23 dimerization and to investigate the extent and nature of the ligand-induced conformational changes. To address the first question, we mutated the so-called 'dimerization arm' within domain II (Fig. 1a) at six sites where analogous mutations are known to disrupt human EGFR dimerization ${ }^{26}$. The resulting sLET-23 $3^{\mathrm{dim}-a r m}$ variant failed to dimerize in SEAUC experiments, sedimenting as a single (monomeric) species of $94 \pm 7 \mathrm{kDa}$ (Fig. 2a and Table 1). Thus, as with ligand-driven human EGFR dimers, the dimerization arm also appears important for constitutive dimerization of sLET-23. Deletion of the invertebrate-specific domain $\mathrm{V}$, at the carboxy terminus of sLET-23 (Fig. 1a), did not prevent dimerization (Fig. 2b and Table 1)-consistent with results mentioned above. By contrast, deleting both domains IV and $\mathrm{V}$ did weaken dimerization substantially (Fig. 2c and Table 1), increasing the value of $K_{\mathrm{d}}^{\mathrm{dim}}$ from sub-micromolar to $30 \pm 13 \mu \mathrm{M}$. Intriguingly, this sLET-23 $\Delta \mathrm{IV} / \mathrm{V}$ variant now displayed ligand-induced dimerization (Fig. $2 \mathrm{c}$ and Table 1) similar to the human or D. melanogaster EGFR extracellular regions, with ligand-binding strengthening dimerization by $\sim 6$-fold (to a $K_{\mathrm{d}}^{\mathrm{dim}}$ of $5 \pm 5 \mu \mathrm{M}$ with LIN-3 ${ }^{\mathrm{EGF}}$ bound).

Ligand-induced structural changes in sLET-23. We used SAXS to monitor the extent of ligand-induced structural changes in the sLET-23 $\Delta \mathrm{V}$ dimer, following approaches that we have used previously for the human and $D$. melanogaster EGFRs ${ }^{18,27}$.

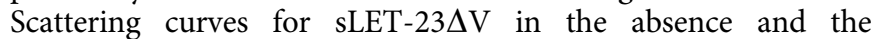
presence of excess ligand were very similar to one another (Fig. 3a,b), although there are small differences in the region beyond $q \sim 0.15 \AA^{-1}$-suggesting ligand-induced conformational changes within the dimer that are very limited in scale. The SAXS-derived radial distance distribution or $P(r)$ curve (Fig. 3c) for unliganded sLET-23AV closely resembles that calculated for the crystallographic dimer of the unliganded D. melanogaster 

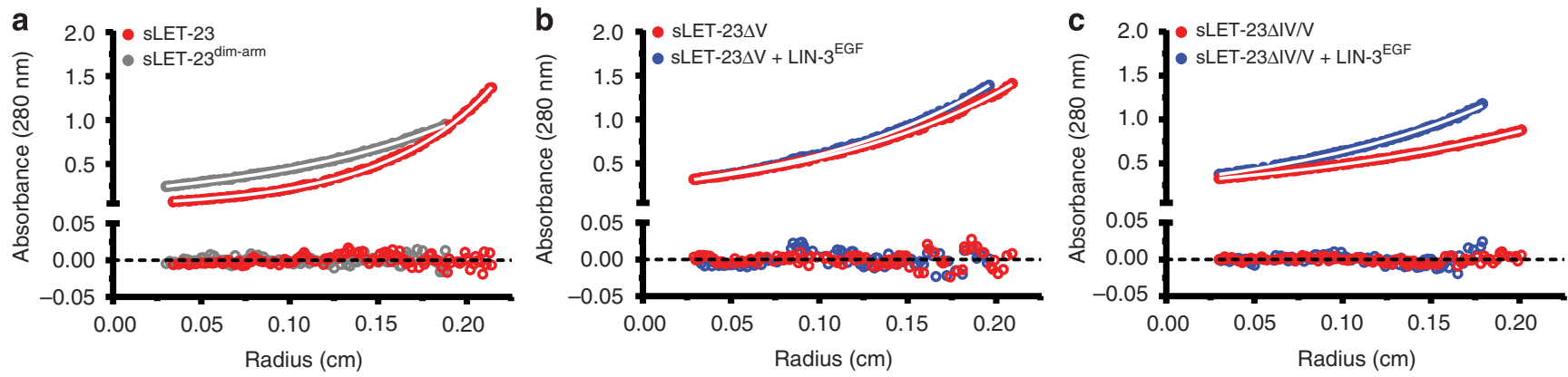

Figure 2 | Determinants of sLET-23 dimerization. (a) Representative SE-AUC data for SLET-23 (red circles) and sLET-23dim-arm (grey circles) collected at $5 \mu \mathrm{M}$ and 10,000 r.p.m. (without added ligand), which fit to single ideal species of $190 \pm 22$ and $94 \pm 7 \mathrm{kDa}$, respectively, across all repeats (see Table 1 and

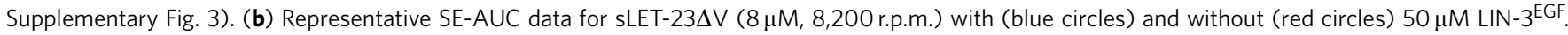
The data fit to single species of $141 \pm 3$ and $153 \pm 27 \mathrm{kDa}$ with and without ligand, respectively (see Table 1 and Supplementary Fig. 3), indicating that ligand binding does not alter oligomerization state. (c) Representative SE-AUC data for sLET-23AIV/V ( $8 \mu \mathrm{M}, 8,200$ r.p.m.) with $60 \mu \mathrm{M} \mathrm{LIN-3}$ EGF (blue circles) or without ligand (red circles). The data show that sLET-23AIV/V is largely monomeric ( $82 \pm 8 \mathrm{kDa}$ ), and that ligand binding promotes dimerization (see Table 1 and Supplementary Fig. 3).
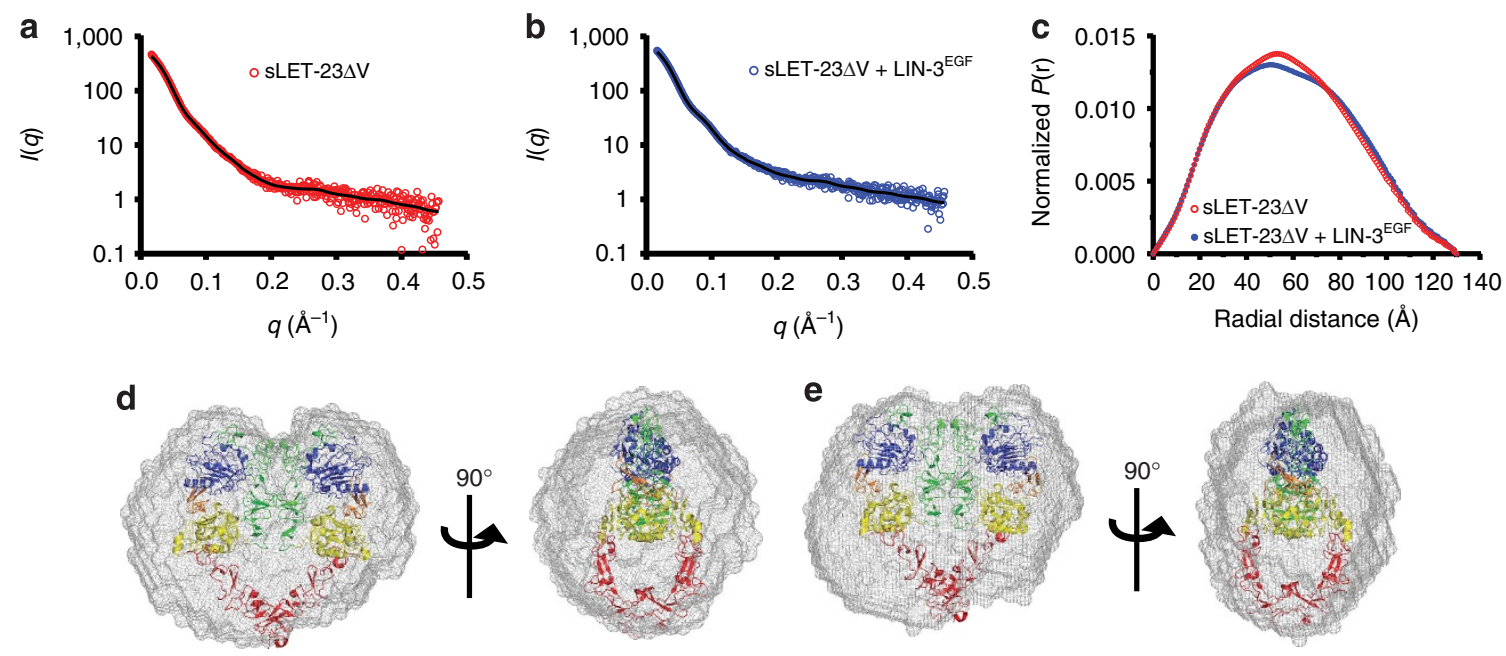

SLET-23AV

SLET-23AV + LIN-3 EGF

Figure 3 | SAXS analysis of ligand-induced conformational changes in sLET-23 $\Delta \mathbf{V}$ dimers. Experimental synchrotron scattering data for $42 \mu \mathrm{M}$ SLET-23 $\mathrm{V}$ in the absence (a, red circles) or presence (b, blue circles) of $100 \mu \mathrm{M}$ added LIN-3EGF, collected at pH 8.0 at MacCHESS (see Supplementary Fig. 4). Data were acquired between $0.0164 \AA^{-1} \leq q \leq 0.456 \AA^{-1}$ and fit with $\mathrm{GNOM}^{36}$ (black line) as described in the Methods. Dilutions to 28 and $14 \mu \mathrm{M}$ did not reveal spurious concentration-dependent effects. (c) SAXS-derived radial distance distributions (or $P(r$ ) curves) for $42 \mu \mathrm{M}$ sLET-23AV with $100 \mu \mathrm{M}$ LIN-3 ${ }^{E G F}$ (blue points) or without added ligand (red points), calculated by inverse Fourier transforms of (a,b). SAXS-derived molecular envelopes, shown as

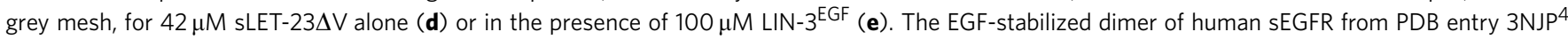
has been manually docked into both envelopes to illustrate the small scale of ligand-induced structural rearrangements. $D_{\max }$ (at $130 \pm 5 \AA$ ) is unchanged on LIN-3 ${ }^{\mathrm{EGF}}$ addition for these studies and the measured value for $R_{\mathrm{g}}$ is also unaltered, at $45.3 \pm 0.5 \AA$.

EGFR extracellular region ${ }^{17}$ (Supplementary Fig. 4a), which is also dimerization arm-mediated. A molecular envelope derived from unliganded sLET-23 $\mathrm{V}$ SAXS data also readily accommodates a model of the EGF-induced sEGFR dimer (Fig. 3d). Thus, our SAXS data indicate that the constitutive sLET-23 dimer resembles a domain II dimerization armmediated dimer of the sort seen in crystal structures of the human and $D$. melanogaster EGFR extracellular regions. Adding excess LIN-3 ${ }^{\text {EGF }}$ results in only small changes to both the $P(r)$ curve (Fig. $3 \mathrm{c}$ and Supplementary Fig. $4 \mathrm{a}$ ) and the SAXS-derived molecular envelope (Fig. 3e), arguing that there are certainly some ligand-induced conformational changes, but that they are subtle. Neither the radius of gyration $\left(R_{\mathrm{g}}\right)$ nor the maximum dimension $\left(D_{\max }\right)$ of the sLET-23 $\Delta \mathrm{V}$ dimer is altered significantly on ligand binding, remaining fixed at $45.3 \pm 0.5$ and $130 \pm 5 \AA$, respectively (Fig. 3c). This contrasts with the $\sim 20 \%$ increase in both of these parameters when EGF binds to (and dimerizes) sEGFR $^{27}$. Nonetheless, LIN-3 ${ }^{\mathrm{EGF}}$ binding does alter the shape of the sLET-23 $\Delta \mathrm{V} P(r)$ curve. It does so in a way that closely resembles the changes seen when comparing the $P(r)$ curve calculated for the unliganded crystallographic dimer of the D. melanogaster EGFR extracellular region (in which the two molecules are 'splayed apart') with that for the liganded D. melanogaster EGFR dimer in which the two molecules are more intimately associated ${ }^{17}$ (Supplementary Fig. 4a). Thus, a comparison of SAXS-derived molecular envelopes for sLET$23 \Delta \mathrm{V}$ with and without bound $\mathrm{LIN}-3^{\mathrm{EGF}}$ (Fig. 3d,e) paints a picture of subtle domain rearrangements that could alter the relative positions of the membrane proximal domains within a LET-23 dimer and allow structural communication of ligand 
occupancy status to the intracellular tyrosine kinase domain in the full-length receptor. Although the resolution of SAXS does not allow us to define these changes precisely, a model of this sort aligns well with recent structure-based suggestions for how IR family receptors function ${ }^{10-13}$. Ligand binding to the IR dimer induces domain rearrangements that in turn alter the position of the membrane proximal domains in a manner that permits productive interactions between the intracellular tyrosine kinase domains for activation. It has been hypothesized that EGFR family members signal similarly ${ }^{8}$ and our data for LET-23 provide the first direct evidence for such ligand-induced structural alterations within preformed EGFR dimers.

\section{Discussion}

Ligand binding drives dimerization and activation of many RTKs, including the human and D. melanogaster EGFRs ${ }^{1,17}$, although it seems clear that these receptors dimerize to some extent even in the absence of ligand ${ }^{8}$. The IR paradigm, and now our data with the C. elegans EGFR LET-23, argue that although RTK dimerization is necessary for activation, it is not sufficient and need not necessarily be ligand induced. A ligand-induced dimerization step in the activation of an RTK may provide an additional level of regulation, and one that is exploited by the human EGFR but not LET-23 in C. elegans. Indeed, certain phosphorylation sites such as threonine 654 in the intracellular juxtamembrane region of human EGFR that have regulatory roles $^{28}$, and may modulate dimerization, are absent in LET-23. Moreover, as in several other RTK families, the single EGFR of C. elegans (or D. melanogaster) is replaced by several orthologues in mammals-allowing signal diversification through receptor heterodimerization ${ }^{29}$. Once dimers are formed, however, the results described here and current models for the IR argue that a precise conformation is required for RTK activation. By belonging to a class of RTKs typically associated with ligandinduced dimerization, yet signalling as a constitutive dimer (without ligand-induced changes in oligomerization state), the C. elegans EGFR, LET-23, appears to represent a 'missing link' between the IR family and other RTKs ${ }^{9}$. Understanding the structural transitions in more detail, and how they are translated into intracellular kinase domain activation, will be a key step in appreciating the significance of the much discussed preformed dimers of EGFRs and other RTKs.

\section{Methods}

Protein expression and purification. Coding regions for sLET-23 variants were subcloned with a C-terminal hexahistidine tag into pFastbac-1 (Invitrogen) for expression in Spodoptera frugiperda (Sf9) cells. Constructs included the native signal sequence and ended at M819 (sLET-23), K645 (sLET-23 $\Delta$ V) or E539 (sLET-23 $\Delta \mathrm{IV} / \mathrm{V})$. 'Dimerization arm' mutations made in domain II of sLET-23 were modelled on those shown to abolish sEGFR dimerization ${ }^{26}$ (F301D, N302A, K304D, G306E, R307A and L308D), and were generated using QuikChange (Stratagene).

DNA encoding the LIN-3 EGF domain (LIN-3 ${ }^{\text {EGF }}$ : residues K148-N206, with numbering from isoform $\mathrm{F} 36 \mathrm{H} 1.4 \mathrm{c}$ on Wormbase) was subcloned into a pMT-V5-His (Invitrogen) variant for expression in D. melanogaster S2 cells as described $^{30}$. The expressed protein contains a BiP signal sequence, followed by a hexahistidine tag, residues 44-76 of D. melanogaster Spitz, and then (after a Factor $\mathrm{Xa}$ cleavage site) $\mathrm{LIN}-3^{\mathrm{EGF}}$, which is released from this fusion protein by Factor Xa cleavage. For expressing full-length LIN-3 (LIN-3 ${ }^{\mathrm{FL}}$ ), DNA encoding its mature extracellular region (isoform $\mathrm{F} 36 \mathrm{H} 1.4 \mathrm{c}$ on Wormbase) was subcloned into pFastbac-1 for Sf9 cell expression, with native signal sequence, a C-terminal hexahistidine tag (following residue S215) and a C23S mutation to prevent possible amino-terminal acylation 31 .

Stable S2 cell pools and recombinant baculoviruses were produced as described ${ }^{17}$. sLET-23 and LIN-3 $3^{\mathrm{FL}}$ were purified from the medium of Sf9 cells that had been infected with baculovirus at a density of $1.5-2.5 \times 10^{6}$ cells per ml. Conditioned medium was harvested 3-4 days post infection and flowed over a Ni-NTA (Qiagen) affinity column. The column was washed with $25 \mathrm{mM} \mathrm{MES,} \mathrm{pH}$ $6.0,150 \mathrm{mM} \mathrm{NaCl}$ (buffer A) and bound protein eluted with an increasing gradient of imidazole in buffer A. For sLET-23, protein-containing fractions were concentrated with an Amicon Ultra 30K concentrator (Millipore), exchanged into buffer A to remove imidazole, and loaded onto an $\mathrm{SO}_{3}^{-}$cation exchange column that was developed using a gradient from $150 \mathrm{mM}$ to $1 \mathrm{M} \mathrm{NaCl}$ in buffer A. Fractions containing sLET-23 were pooled, concentrated and applied to a Superose 6 10/300 GL column (GE Healthcare) equilibrated in $10 \mathrm{mM}$ Hepes, pH 8.0, $150 \mathrm{mM} \mathrm{NaCl}$ (buffer B). The final purity of sLET-23 was $>95 \%$ by Coomassiestained SDS-PAGE. Following Ni-NTA affinity chromatography, LIN-3 ${ }^{\mathrm{FL}}$ was concentrated and purified further using a Superose 12 column (GE Healthcare) equilibrated in buffer B. Human sEGFR was produced exactly as described ${ }^{32}$.

LIN-3 ${ }^{\text {EGF }}$ was purified from $500 \mathrm{ml}$ of S2 cells grown to $\sim 4-6 \times 10^{6}$ cells per $\mathrm{ml}$ and induced with $750 \mu \mathrm{M} \mathrm{CuSO}_{4}$ for 4 days. Conditioned medium was dialysed in $24 \mathrm{l}$ of $10 \mathrm{mM}$ Hepes, $\mathrm{pH} 8.0,100 \mathrm{mM} \mathrm{NaCl}$ for $16 \mathrm{~h}$ at $4{ }^{\circ} \mathrm{C}$ and then bound to a 2-ml Ni-NTA column. After washing with buffer A, bound protein was eluted with an increasing gradient of imidazole in buffer A. LIN-3 ${ }^{\mathrm{EGF}}$ was concentrated, exchanged into buffer A to remove imidazole, and purified by cation exchange as for sLET-23. LIN-3 ${ }^{\mathrm{EGF}}$ was then exchanged into $10 \mathrm{mM}$ Hepes, $\mathrm{pH} 7.0,100 \mathrm{mM}$ $\mathrm{NaCl}, 2 \mathrm{mM} \mathrm{CaCl}_{2}$, concentrated to $1 \mathrm{mg} \mathrm{ml}^{-1}$ and cleaved overnight at $4{ }^{\circ} \mathrm{C}$ with $20 \mu \mathrm{g}$ Factor Xa. Cleaved protein was then applied to a Ni-NTA column to remove the His-tagged $\mathrm{N}$ terminus and the $\mathrm{LIN}-3^{\mathrm{EGF}}$-containing flow-through was concentrated and further purified by size exclusion using a Superdex peptide column (GE Healthcare) equilibrated in buffer B.

Size exclusion and MALS experiments. Size-exclusion chromatography experiments employed a Superose 6 10/300 GL column (GE Healthcare) equilibrated in buffer B. The column was calibrated with thyroglobulin $(670 \mathrm{kDa}), \gamma$-globulin $(158 \mathrm{kDa})$, ovalbumin $(44 \mathrm{kDa})$, myoglobin $(17 \mathrm{kDa})$ and vitamin $\mathrm{B}_{12}(1.4 \mathrm{kDa})$. Experimental proteins were loaded at $4 \mathrm{mg} \mathrm{ml}^{-1}$ in buffer B and molecular masses were determined using a DAWN HELEOS II MALS with an on-line Optilab T-rEX interferometric refractometer (Wyatt Technology). The refractive index increment $(\mathrm{d} n / \mathrm{dc})$ was estimated as 0.185 and the molecular mass of each protein within defined chromatographic peaks was calculated using ASTRA software version 6.0 (Wyatt Technology) as recommended by the manufacturer.

Sedimentation equilibrium analytical ultracentrifugation. SE-AUC experiments employed an XL-A analytical ultracentrifuge with an An-60 Ti rotor (Beckman Coulter, Fullerton, CA). Samples of sLET-23 proteins ranging from 1 to $8 \mu \mathrm{M}$ in buffer B were analysed with and without addition of $50-70 \mu \mathrm{M} \mathrm{LIN}-3^{\mathrm{EGF}}$ in six-channel charcoal-Epon cells as described previously for our studies of human $\mathrm{EGFR}^{33}$, at $20^{\circ} \mathrm{C}$, at three speeds (between 5,000 and 12,500 r.p.m.), detecting at 280,290 and $295 \mathrm{~nm}$. Radial positions are plotted in figures as $r-r_{0}$, where $r_{0}$ is the meniscus. The partial specific volume of sLET-23 was estimated as $0.71 \mathrm{mlg}^{-1}$, solvent density as $1.003 \mathrm{~g} \mathrm{ml}^{-1}$ and buffer viscosity as $1.0204 \times 10^{-2} \mathrm{cPoise}$. For each sample, equilibrium data at three speeds were analysed as a group with HeteroAnalysis (v1.1.57, written by J. Cole and J. Lary, University of Connecticut) and were fit as a single ideal species. To estimate $K_{\mathrm{d}}^{\mathrm{dim}}$ for sLET-23 $\Delta \mathrm{IV} / \mathrm{V}$, data from three concentrations were analysed as a group and fit in HeteroAnalysis to a model describing simple receptor dimerization, floating only $K_{\mathrm{d}}^{\mathrm{dim}}$. Errors quoted in the text are means \pm s.d. for 3-18 independent measurements.

Small angle X-ray scattering. Home-source data were recorded at $4{ }^{\circ} \mathrm{C}$ on a Rigaku PSAXS S-Max3000 pinhole camera system with a Rigaku 007HF rotating anode source and a Rigaku $300 \mathrm{~mm}$ wire grid ASM DTR 200 detector, with 20-80 min exposures. Protein concentration ranged from 1.2 to $5 \mathrm{mg} \mathrm{ml}^{-1}$ in buffer B ( $\mathrm{pH} 8.0$ ). EGF was added to sEGFR samples at a 1.2-fold molar excess and LIN-3 ${ }^{\mathrm{EGF}}$ was added in larger excesses (adding $61-110 \mu \mathrm{M}$ ), to reach $>90 \%$ saturation of sLET- $23 \Delta \mathrm{V}$. In the latter case, LIN-3 $3^{\mathrm{EGF}}$ at the concentration estimated to be unbound (based on the $K_{\mathrm{d}}$ values reported in Supplementary Fig. 2) was included in the buffer blank for subtraction. Home-source data were reduced using SAXSGui v2.05.02 (Rigaku America \& JJ X-Ray Systems ApS, Lyngby, Denmark) and matching buffers were subtracted using PRIMUS ${ }^{34}$, to yield the final scattering profile in which intensity $(I)$ is plotted as a function of $q(4 \pi \sin \theta / \lambda$, where $2 \theta$ is the scattering angle). Synchrotron data were acquired at CHESS beamline F1, operating at $12.686 \mathrm{keV}$. Samples were protected from radiation damage by the addition of $5 \% \mathrm{v} / \mathrm{v}$ glycerol to buffer B and oscillation in the X-ray beam using a computer-controlled syringe pump. The X-ray beam was collimated to $250 \times 250 \mu \mathrm{m}^{2}$ and images were collected with a Dectris Pilatus $100 \mathrm{~K}-\mathrm{S}$ detector, with sample-to-detector distance of $1,140 \mathrm{~mm}$ and momentum transfer interval of $0.0164 \AA^{-1}<q<0.456 \AA^{-1}$. Ten exposures of $4 \mathrm{~s}$ were averaged and the data were subtracted and reduced using BioXTAS RAW software ${ }^{35}$

All samples were monodisperse as evidenced by linear Guinier regions where $q R_{\mathrm{g}} \leq 1.3$ and Kratky plots showed no signs of radiation-induced denaturation. $P(r)$ curves and values for $I(0), R_{\mathrm{g}}$ and $D_{\max }$ were obtained using the programme $\mathrm{GNOM}^{36}$. $R_{\mathrm{g}}$ values calculated with GNOM were consistent with those estimated from the Guinier region, and the total estimate for each GNOM run was better than 0.90 . $I(0) / c$ measurements (Fig. 1d) were all performed using our home source, taking advantage of beam stability. Measured intensities were converted to absolute units during data reduction using beam transmission corrections and the scattering from glassy carbon. $I(0)$ values were normalized by mass concentration, and parallel measurements using reference proteins of known mass were consistent 
with literature values ${ }^{37}$ : lysozyme $(14.6 \mathrm{kDa})$, ovalbumin $(44.3 \mathrm{kDa})$ and glucose isomerase $(173 \mathrm{kDa})$. The structural information described in Fig. 3 are all from data collected at CHESS. Using synchrotron scattering data up to $q_{\max }=0.3 \AA^{-1}$, low-resolution molecular envelopes (Fig. 3d,e) were constructed by averaging 20 independent DAMMIN ${ }^{38}$ runs (with no imposed symmetry) using the programme DAMAVER ${ }^{39}$. Mean normalized spatial discrepancy values for sLET-23AV and sLET-23 $\Delta \mathrm{V}$ with bound LIN-3 $3^{\mathrm{EGF}}$ were 0.76 and 0.77 , respectively. pH-dependent differences in SAXS parameters seen for unliganded sLET-23 $\Delta \mathrm{V}$ in our homesource experiments (see Supplementary Fig. $4 \mathrm{~b}, \mathrm{c}$ ) were not observed in experiments performed at CHESS. Molecular mass estimates computed at CHESS for sLET-23 $\Delta \mathrm{V}$ (derived from comparison of $I(0) / \mathrm{c}$ with lysozyme and glucose isomerase standards) were also consistent with constitutive sLET- $23 \Delta \mathrm{V}$ dimerization.

Analysis of LET-23 activation. D. melanogaster S2 cells were chosen as a null background, because no $D$. melanogaster EGFR activation can be detected when these cells are treated with its activating ligands ${ }^{40}$ and, in addition, we cannot detect binding of LIN-3 to the D. melanogaster EGFR extracellular region. S2 cells stably transfected with full-length LET-23 (containing a C-terminal hexahistidine tag) were serum starved overnight. LET-23 expression was induced for $3.5 \mathrm{~h}$ with $60 \mu \mathrm{M}$ $\mathrm{CuSO}_{4}$ and LIN-3 ${ }^{\mathrm{EGF}}$ was added at the indicated concentrations for $10 \mathrm{~min}$ on ice, in PBS containing $0.5 \%(\mathrm{w} / \mathrm{v})$ BSA. Cells were then lysed in ice-cold $25 \mathrm{mM}$ Tris, $\mathrm{pH}$ $7.5,150 \mathrm{mM} \mathrm{NaCl}, 1 \%$ Nonidet P40 containing protease and phosphatase inhibitors. His-tagged LET-23 was enriched from clarified lysates by Ni-NTA affinity precipitation and samples were analysed by western blotting using phosphotyrosine $\left(\alpha\right.$-pY20; Santa Cruz Biotechnology) and $\mathrm{His}_{5}$ (Qiagen) antibodies at dilutions of $1: 500$ and $1: 1,000$, respectively, followed by incubation with relevant secondary antibodies conjugated to fluorescent dyes (LI-COR Biosciences, Lincoln, NE) for detection at 680 and $800 \mathrm{~nm}$ using LI-COR Odyssey imaging.

Surface plasmon resonance. Surface plasmon resonance experiments employed a Biacore 3,000 instrument. LIN-3 $3^{\mathrm{FL}}$ and LIN- $3^{\mathrm{EGF}}$ were immobilized on a Biacore CM5 biosensor chip using amine coupling as described ${ }^{32}$. Purified sLET-23 was injected at the indicated concentrations at $5 \mu \mathrm{min}^{-1}$ for $9 \mathrm{~min}$ (sufficient for binding to reach steady state), in degassed $10 \mathrm{mM}$ Hepes (pH 8.0), $150 \mathrm{mM} \mathrm{NaCl}$, $3 \mathrm{mM}$ EDTA and $0.005 \%$ Surfactant P-20 at room temperature. The final steadystate signal was background corrected by subtraction of the signal obtained with the control surface. Values were plotted against [sLET-23 variant] and fit to a simple single-site saturation-binding model. Between injections, the surface was regenerated using a $10-\mu \mathrm{l}$ injection of $10 \mathrm{mM}$ sodium acetate, $\mathrm{pH} 5.0,1 \mathrm{M} \mathrm{NaCl}$.

\section{References}

1. Lemmon, M. A. \& Schlessinger, J. Cell signaling by receptor tyrosine kinases. Cell 141, 1117-1134 (2010).

2. Schlessinger, J. Signal transduction by allosteric receptor oligomerization. Trends Biochem. Sci. 13, 443-447 (1988).

3. Williams, L. T. Signal transduction by the platelet-derived growth factor receptor. Science 243, 1564-1570 (1989).

4. Lu, C. et al. Structural evidence for loose linkage between ligand binding and kinase activation in the epidermal growth factor receptor. Mol. Cell. Biol. 30, 5432-5443 (2010).

5. Mi, L. Z. et al. Simultaneous visualization of the extracellular and cytoplasmic domains of the epidermal growth factor receptor. Nat. Struct. Mol. Biol. 18, 984-989 (2011).

6. Bessman, N. J., Freed, D. M. \& Lemmon, M. A. Putting together structures of epidermal growth factor receptors. Curr. Opin. Struct. Biol. 29, 95-101 (2014).

7. Endres, N. F. et al. Conformational coupling across the plasma membrane in activation of the EGF receptor. Cell 152, 543-556 (2013).

8. Maruyama, I. N. Mechanisms of activation of receptor tyrosine kinases: monomers or dimers. Cells 3, 304-330 (2014).

9. De Meyts, P. The insulin receptor: a prototype for dimeric, allosteric membrane receptors? Trends Biochem. Sci. 33, 376-384 (2008).

10. McKern, N. M. et al. Structure of the insulin receptor ectodomain reveals a folded-over conformation. Nature 443, 218-221 (2006).

11. Kavran, J. M. et al. How IGF-1 activates its receptor. eLife 3, e03772 (2014).

12. Menting, J. G. et al. How insulin engages its primary binding site on the insulin receptor. Nature 493, 241-245 (2013).

13. Ward, C. W., Menting, J. G. \& Lawrence, M. C. The insulin receptor changes conformation in unforeseen ways on ligand binding: sharpening the picture of insulin receptor activation. Bioessays 35, 945-954 (2013).

14. Aroian, R. V., Koga, M., Mendel, J. E., Ohshima, Y. \& Sternberg, P. W. The let-23 gene necessary for Caenorhabditis elegans vulval induction encodes a tyrosine kinase of the EGF receptor subfamily. Nature 348, 693-699 (1990).

15. Hill, R. J. \& Sternberg, P. W. The gene lin-3 encodes an inductive signal for vulval development in C. elegans. Nature 358, 470-476 (1992).
16. Lemmon, M. A. et al. Two EGF molecules contribute additively to stabilization of the EGFR dimer. EMBO J. 16, 281-294 (1997).

17. Alvarado, D., Klein, D. E. \& Lemmon, M. A. Structural basis for negative cooperativity in growth factor binding to an EGF receptor. Cell 142, 568-579 (2010).

18. Alvarado, D., Klein, D. E. \& Lemmon, M. A. ErbB2 resembles an autoinhibited invertebrate epidermal growth factor receptor. Nature 461, 287-291 (2009).

19. Schlessinger, J. in Physical Chemistry of Cell Surface Events in Cellular Regulation. (eds De Lisi, C. \& Blumenthal, R.) 89-118 (Elsevier, 1979).

20. Red Brewer, M. et al. The juxtamembrane region of the EGF receptor functions as an activation domain. Mol. Cell 34, 641-651 (2009).

21. Jura, N. et al. Mechanism for activation of the EGF receptor catalytic domain by the juxtamembrane segment. Cell 137, 1293-1307 (2009).

22. Mendrola, J. M., Berger, M. B., King, M. C. \& Lemmon, M. A. The single transmembrane domains of ErbB receptors self-associate in cell membranes. J. Biol. Chem. 277, 4704-4712 (2002).

23. Grasberger, B., Minton, A. P., DeLisi, C. \& Metzger, H. Interaction between proteins localized in membranes. Proc. Natl Acad. Sci. USA 83, 6258-6262 (1986).

24. Macdonald-Obermann, J. L. \& Pike, L. J. Different epidermal growth factor (EGF) receptor ligands show distinct kinetics and biased or partial agonism for homodimer and heterodimer formation. J. Biol. Chem. 289, 26178-26188 (2014).

25. Golembo, M., Yarnitzky, T., Volk, T. \& Shilo, B. Z. Vein expression is induced by the EGF receptor pathway to provide a positive feedback loop in patterning the Drosophila embryonic ventral ectoderm. Genes Dev. 13, 158-162 (1999).

26. Garrett, T. P. J. et al. Crystal structure of a truncated epidermal growth factor receptor extracellular domain bound to transforming growth factor alpha. Cell 110, 763-773, 2002).

27. Dawson, J. P., Bu, Z. \& Lemmon, M. A. Ligand-induced structural transitions in ErbB receptor extracellular domains. Structure 15, 942-954 (2007).

28. Thiel, K. W. \& Carpenter, G. Epidermal growth factor receptor juxtamembrane region regulates allosteric tyrosine kinase activation. Proc. Natl Acad. Sci. USA 104, 19238-19243 (2007).

29. Yarden, Y. \& Sliwkowski, M. X. Untangling the ErbB signalling network. Nat. Rev. Mol. Cell Biol. 2, 127-137 (2001).

30. Klein, D. E., Stayrook, S. E., Shi, F., Narayan, K. \& Lemmon, M. A. Structural basis for EGFR ligand sequestration by Argos. Nature 453, 1271-1275 (2008).

31. Miura, G. I. et al. Palmitoylation of the EGFR ligand Spitz by Rasp increases Spitz activity by restricting its diffusion. Dev. Cell 10, 167-176 (2006).

32. Ferguson, K. M., Darling, P. J., Mohan, M. J., Macatee, T. L. \& Lemmon, M. A. Extracellular domains drive homo- but not hetero-dimerization of erbB receptors. EMBO J. 19, 4632-4643 (2000).

33. Dawson, J. P. et al. Epidermal growth factor receptor dimerization and activation require ligand-induced conformational changes in the dimer interface. Mol. Cell. Biol. 25, 7734-7742 (2005).

34. Konarev, P. V., Volkov, V. V., Sokolova, A. V., Koch, M. H. J. \& Svergun, D. I PRIMUS: a Windows PC-based system for small-angle scattering data analysis. J. Appl. Crystallogr. 36, 1277-1282 (2003).

35. Nielsen, S. S. et al. BioXTAS RAW, a software program for high-throughput automated small-angle X-ray scattering data reduction and preliminary analysis. J. Appl. Crystallogr. 42, 959-964 (2009).

36. Semenyuk, A. V. \& Svergun, D. I. Gnom - a program package for small-angle scattering data-processing. J. Appl. Crystallogr. 24, 537-540 (1991).

37. Mylonas, E. \& Svergun, D. I. Accuracy of molecular mass determination of proteins in solution by small-angle X-ray scattering. J. Appl. Crystallogr. 40, S245-S249 (2007).

38. Svergun, D. I. Restoring low resolution structure of biological macromolecules from solution scattering using simulated annealing. Biophys. J. 76, 2879-2886 (1999).

39. Volkov, V. V. \& Svergun, D. I. Uniqueness of ab-initio shape determination in small-angle scattering. J. Appl. Crystallogr. 36, 860-864 (2003).

40. Klein, D. E., Nappi, V. M., Reeves, G. T., Shvartsman, S. Y. \& Lemmon, M. A. Argos inhibits epidermal growth factor receptor signalling by ligand sequestration. Nature 430, 1040-1044 (2004).

\section{Acknowledgements}

We thank members of the Lemmon and Ferguson laboratories for advice and critical reading of this manuscript, and Steve Stayrook and Richard Gillilan (CHESS) for assistance with the SAXS measurements. Synchrotron SAXS data were collected at MacCHESS beamline F1. CHESS is supported by the NSF and NIH/NIGMS via NSF award DMR-0936384 and the MacCHESS resource is supported by NIH/NIGMS award GM-103485. This work was supported by a grant from the National Institutes of Health R01-CA079992 to M.A.L. and Postdoctoral Fellowship F32-GM109688 from the National Institutes of Health to D.M.F. 


\section{Author contributions}

D.M.F., D.A. and M.A.L. designed the study and analysed the resulting data. D.M.F. and D.A. carried out the experiments under the supervision of M.A.L., and D.M.F. and M.A.L. wrote the paper.

\section{Additional information}

Supplementary Information accompanies this paper at http://www.nature.com/ naturecommunications

Competing financial interests: The authors declare no competing financial interests.
Reprints and permission information is available online at http://npg.nature.com/ reprintsandpermissions/

How to cite this article: Freed, D. M. et al. Ligand regulation of a constitutively dimeric EGF receptor. Nat. Commun. 6:7380 doi: 10.1038/ncomms8380 (2015).

(c) (i) This work is licensed under a Creative Commons Attribution 4.0 cc International License. The images or other third party material in this article are included in the article's Creative Commons license, unless indicated otherwise in the credit line; if the material is not included under the Creative Commons license, users will need to obtain permission from the license holder to reproduce the material. To view a copy of this license, visit http://creativecommons.org/licenses/by/4.0/ 